\title{
Effect of treatment modality on post-operative renal function in patients with upper tract urothelial
} cancer

\begin{abstract}
Background: While endoscopic management (EM) and distal ureterectomy (DU) provide renal-sparing alternatives to radical nephroureterectomy $(\mathrm{NU})$, the effects on renal function have never directly been compared.

Objectives: While NU is the "gold standard" for treating upper tract urothelial carcinoma (UTUC), EM and DU have become popular for certain lesions. However, the true effect of these procedures on renal function has been under-studied. We analyzed the impact of EM, DU, and NU on post-operative renal function in both the short- and long-term intervals.

Methods: Patients undergoing EM, DU, or NU for UTUC were included. Clinicopathologic information was collected. eGFR values were calculated via MDRD formula. Absolute change in eGFR was compared amongst groups. Linear mixed model regression analysis was used to determine the impact of procedure type on post-operative eGFR.

Results: 273 patients underwent 325 procedures from 1991 to 2012. In both shortand long-term intervals, post-operative eGFR was significantly worse following NU compared to EM. For the long-term interval, this difference remained while controlling for age, race, gender, pathologic stage, pre-operative hydronephrosis, and having a solitary kidney $($ all $\mathrm{p}<0.05)$. Post-operative eGFR change was not significantly impacted by DU compared to EM.

Conclusion: $\mathrm{EM}$ is superior to $\mathrm{NU}$ and equivalent to $\mathrm{DU}$ in preserving post-operative eGFR for UTUC patients. The non-inferiority of DU in this outcome validates its use as a kidney-sparing alternative alongside EM. The effects of these treatment modalities on renal function should be considered with oncologic outcomes when deciding management for these patients.
\end{abstract}

Keywords: upper tract urothelial carcinoma, endoscopic management, distal ureterectomy, renal function, conservative management
Volume I Issue I - 2014

\author{
Srinath Kotamarti,' Byron P Dubow,' \\ Arindam Roy Choudhury, ${ }^{2}$ Matthew R \\ Danzig,' Mantu Gupta,' James M McKiernan,' \\ G Joel DeCastro,' Sven Wenske' \\ 'Department of Urology, Columbia University College of \\ Physicians and Surgeons, USA \\ ${ }^{2}$ Columbia University Mailman School of Public Health, USA
}

Correspondence: Srinath Kotamarti, Department of Urology, Herbert Irving Cancer Center, Columbia University College of Physicians and Surgeons, New York, NY 10032, USA, Tel 9087528962,Email sountp@hotmail.com

Received: October 01, 2014 | Published: October 17, 2014
Abbreviations: UTUC, upper tract urothelial carcinoma; eGFR, estimated GFR; MDRD, modified diet in renal disease; EM, endoscopic management; DU, distal ureterectomy; NU, nephroureterectomy; CKD, chronic kidney disease; ANOVA, analysis of variance; WHO, world health organization

\section{Introduction}

Upper tract urothelial carcinoma (UTUC) is a rare, aggressive disease comprising approximately $5-6 \%$ of all urothelial carcinomas. ${ }^{1}$ Compared to patients with lower tract urothelial carcinomas, patients with UTUC usually present with more invasive and higher grade disease. ${ }^{2}$ The increased prevalence over time of comorbidities such as chronic kidney disease can further complicate the presentation and management of UTUC. ${ }^{3,4}$ Historically, radical nephroureterectomy (NU) has been the gold standard for treatment of this disease. ${ }^{5}$

Considering that advanced UTUC disease is practically incurable, treatment before progression to advanced stage is paramount. ${ }^{6-8}$ Distal ureterectomy was developed as an alternative to NU for distal ureteral lesions and shown to have acceptable oncologic and functional outcomes. ${ }^{9,10}$ Endoscopic management, including both laser ablation and fulguration, has been used increasingly for certain lesions. ${ }^{5}$ Conservative alternatives are renal-sparing, less invasive, and traditionally pursued in patients with a solitary kidney, bilateral disease, or disease that is low grade or low volume. ${ }^{1,11}$ In addition, the delay to radical surgery caused by undergoing ureteroscopic procedures has been shown not to affect oncologic outcomes after NU. ${ }^{12}$ EM has been shown to be effective in cases of unifocal, $<2 \mathrm{~cm}$, low grade, and low stage disease; however, strict adherence to follow up protocol is necessary. ${ }^{13}$

Though EM and DU are considered kidney-sparing approaches, the effects on renal function are understudied. A study by Fajkovic and colleagues compared 20 patients receiving EM to 178 patients receiving NU and found worse post-operative kidney function after NU. ${ }^{14}$ Whether conservative management confers a functional advantage in both the short-term as well as long-term intervals has also been underexplored. The goal of our analysis was to determine the extent to which conservative management affects both immediate post-operative and long-term renal function compared to radical NU or DU at our institution.

\section{Patients and methods}

The IRB-approved Columbia University Medical Center Urologic 
Oncology database was queried to identify all patients who underwent EM, DU, or NU for UTUC from $1 / 1980$ to $12 / 2012$. Patient demographics, perioperative and pathologic data were collected from a retrospective chart review.

Renal functional outcomes were measured by change in estimated GFR (eGFR), from one month pre-operatively to the post-operative period. The Modified Diet in Renal Disease (MDRD) formula was used to calculate eGFR. The absolute change in eGFR and the percent change in eGFR were then determined. UTUC procedures that occurred after a cystectomy were excluded due to the possible confounding influences this surgery would have on eGFR. Pathologic verification was obtained via ureteroscopic cold-cup biopsy and surgical pathology at time of NU or DU. Pathologic grading was obtained when available and normalized in accordance with 2004 WHO criteria.

EM for these patients consisted of retrograde ureteroscopy with tumor resection or laser ablation. It must be noted that patients undergoing EM each had multiple ureteroscopic procedures during the course of their conservative management. Procedures were excluded if records could not be obtained during our database search or if no corresponding creatinine values were found. If patients underwent procedures outside of our institution, those specific procedures were not included in this study. NU was performed with a bladder cuff.

Patients underwent standard follow-up with laboratory tests for renal function, as well as upper tract imaging via CT urography or retrograde ureteropyelography as needed. The immediate impact of each procedure type was assessed, with the short-term interval defined as follows: For EM, the date of first negative ureteroscopic assessment after initial EM, or the date of management change to DU or NU; for DU and NU, the interval lasted till one month post-operatively. On the other hand, the long-term follow-up interval was defined as the length of time between the date of surgery and the latest creatinine value available. Patients undergoing multiple procedures were analyzed for change in their renal function until their next procedure took place.

For analysis of the immediate impact of each procedure type, the changes in eGFR after each procedure during the short-term interval were compared using ANOVA analysis and the Student's T test. To analyze the long-term interval, absolute change in eGFR $(\triangle \mathrm{eGFR})$ from the pre-operative value to the latest value available was compared between procedure types via univariate and multivariate linear mixed model regression analyses.

All statistics were performed with R (R Foundation for Statistical Computing, Vienna, Austria). All p-values were the result of twosided tests, and a p-value of less than 0.05 was considered statistically significant.

\section{Results}

There were 273 patients undergoing a total of 325 procedures that met inclusion criteria. Demographic, clinical, and outcome data for patients in each of the three procedure groups is described in Table 1.

Average pre-operative eGFR for EM, DU, and NU were $62.80 \mathrm{~mL} /$ $\min / 1.73 \mathrm{~m}^{2} \quad(\mathrm{SD}=25.4), \quad 62.82 \mathrm{~mL} / \mathrm{min} / 1.73 \mathrm{~m}^{2} \quad(\mathrm{SD}=22.9)$, and $61.59 \mathrm{~mL} / \mathrm{min} / 1.73 \mathrm{~m}^{2}(\mathrm{SD}=21.0)$, respectively; these values were not statistically different on ANOVA analysis. No patients among the difference procedure types had pre-operative Chronic Kidney Disease (CKD) stage 5 renal functions. Short-term average eGFR changes for each procedure type were $-2.97 \mathrm{~mL} / \mathrm{min} / 1.73 \mathrm{~m}^{2}(\mathrm{SD}=16.8),-1.06 \mathrm{~mL} /$ $\mathrm{min} / 1.73 \mathrm{~m}^{2} \quad(\mathrm{SD}=23.1)$, and $-17.38 \mathrm{~mL} / \mathrm{min} / 1.73 \mathrm{~m}^{2} \quad(\mathrm{SD}=20.1)$, respectively. There was a significantly less negative average eGFR change during the short-term follow-up interval in patients undergoing EM compared to those undergoing $\mathrm{NU}(\mathrm{p}<0.001)$ and in patients undergoing DU compared to those undergoing NU $(p<0.001)$. No significant difference was found in average eGFR change between patients undergoing EM and those undergoing DU (Table 2).

The average long-term $\triangle \mathrm{eGFR}$ for EM, DU, and NU was $-4.69 \mathrm{~mL} /$ $\mathrm{min} / 1.73 \mathrm{~m}^{2} \quad(\mathrm{SD}=21.5), \quad-3.70 \mathrm{~mL} / \mathrm{min} / 1.73 \mathrm{~m}^{2} \quad(\mathrm{SD}=21.8)$, and $-15.37 \mathrm{~mL} / \mathrm{min} / 1.73 \mathrm{~m}^{2}(\mathrm{SD}=23.0)$, respectively (Table 1$)$. Fourteen patients $(6.25 \%)$ of NU patients ended up with CKD Stage 5 renal function compared to 1 patient $(1.96 \%)$ for EM and 0 patients for DU (Table 1). NU was further associated with a significantly larger absolute decrease in eGFR during the long-term interval compared to EM on univariate regression analysis $(\mathrm{p}=0.009)$. This significance remained after controlling for age, tumor stage, hydronephrosis, and solitary kidney status $(\mathrm{p}=0.03)$. In contrast, DU vs. EM was not a significant predictor of absolute change in long-term eGFR on either univariate or multivariate analysis (Table 3).

Table I Characteristics by procedure type ${ }^{\mathrm{a}}$

\begin{tabular}{|c|c|c|c|c|c|c|}
\hline & EM & & DU & & NU & \\
\hline Patients (N) & 39 & & 53 & & 222 & \\
\hline Procedures (N) & 48 & & 53 & & 224 & \\
\hline Median Age (years, range) & 71 & $(40-88)$ & 71 & $(40-86)$ & 71 & $(3 \mid-87)$ \\
\hline Males (N) & 33 & & 40 & & 140 & \\
\hline Females $(\mathrm{N})$ & 22 & & 13 & & 84 & \\
\hline Patients with a Solitary Kidney Prior (N) & I & & 3 & & 1 & \\
\hline \multicolumn{7}{|l|}{ Laterality $(\mathrm{N})$ (I case unknown) } \\
\hline Right & 26 & & 19 & & 109 & \\
\hline Left & 22 & & 32 & & 112 & \\
\hline Bilateral & 0 & & 2 & & 2 & \\
\hline \multicolumn{7}{|l|}{ Surgical Modality (N) } \\
\hline Endoscopic & 48 & & 0 & & 0 & \\
\hline
\end{tabular}


Table Continued..

\begin{tabular}{|c|c|c|c|c|c|c|}
\hline & EM & & DU & & NU & \\
\hline Open & 0 & & 52 & & 112 & \\
\hline Laparoscopic & 0 & & 0 & & 53 & \\
\hline Laparoscopic Nephrectomy with Open Ureterectomy & 0 & & 0 & & 52 & \\
\hline Laparoscopic Converted to Open & 0 & & 0 & & 2 & \\
\hline Robot-Assisted Laparoscopic & 0 & & I & & 5 & \\
\hline \multicolumn{7}{|l|}{ Pathologic Stage (N) } \\
\hline $\mathrm{Ta}$ & 6 & & 3 & & 71 & \\
\hline Tis & 29 & & 17 & & 12 & \\
\hline TI & 5 & & 6 & & 44 & \\
\hline $\mathrm{T} 2$ & 7 & & 2 & & 16 & \\
\hline T3 & 0 & & 7 & & 64 & \\
\hline $\mathrm{T} 4$ & I & & 7 & & 13 & \\
\hline \multicolumn{7}{|l|}{ Pathologic Grade (when described) (N) } \\
\hline High Grade & 19 & & 24 & & 136 & \\
\hline Low Grade & 23 & & 16 & & 70 & \\
\hline Patients with a History of Upper Tract BCG/MMC Course & 6 & & 1 & & 12 & \\
\hline Pre-Op Creatinine (mg/dl, range) & 1.2 & $(0.5-2.5)$ & I.I & $(0.6-3.4)$ & I.I & $(0.5-3.6)$ \\
\hline eGFR Decrease from Pre-Op to Long-Term Post-Op (mL/min/I.73m2) (average, SD) & 4.69 & \pm 21.5 & 3.7 & \pm 21.8 & 15.4 & \pm 23.0 \\
\hline Patients with Post-Op CKD Stage 5 & I & & 0 & & & 14 \\
\hline Long-term follow up (months, range) & 19.5 & $(I-185)$ & 21 & $(I-230)$ & 22 & $(1-232)$ \\
\hline
\end{tabular}

aAbbreviations: EM, endoscopic management; DU, distal ureterectomy; NU, nephroureterectomy; CKD, chronic kidney disease

Table 2 Analysis for short-term post-operative renal function ${ }^{\mathrm{a}, \mathrm{b}}$

\begin{tabular}{|c|c|c|c|c|}
\hline \multicolumn{5}{|l|}{ Descriptive statistics } \\
\hline & EM & DU & NU & Overall \\
\hline Mean Pre-Op eGFR (mL/min/I.73m²) & $62.80 \pm 25.4$ & $62.82 \pm 22.9$ & $61.59 \pm 21.0$ & $61.98 \pm 22.0$ \\
\hline Mean Post-Op eGFR(mL/min/I.73m²) & $60.80 \pm 23.6$ & $63.31 \pm 21.1$ & $44.36 \pm 13.9$ & $49.89 \pm 18.8$ \\
\hline Mean eGFR change $\left(\mathrm{mL} / \mathrm{min} / 1.73 \mathrm{~m}^{2}\right)$ & $-2.97 \pm 16.8$ & $-1.06 \pm 23.1$ & $-17.38 \pm 20.1$ & $-12.57 \pm 21.3$ \\
\hline \multicolumn{5}{|l|}{ Student's T test } \\
\hline & EM vs. NU & EM vs. DU & DU vs. NU & \\
\hline Pre-Op eGFR & 0.72 & 0.99 & 0.71 & \\
\hline Post-Op eGFR & $<0.001$ & 0.59 & $<0.001$ & \\
\hline eGFR change & $<0.001$ & 0.65 & $<0.00$ I & \\
\hline ANOVA & p value & & & \\
\hline Pre-Op eGFR & 0.9 & & & \\
\hline Post-Op eGFR & $<0.001$ & & & \\
\hline eGFR change & $<0.001$ & & & \\
\hline
\end{tabular}

${ }^{a}$ Values are presented as mean with SD or $\mathrm{P}$ value

${ }^{b}$ Abbreviations: EM, endoscopic management; DU, distal ureterectomy; NU, nephroureterectomy 
Table 3 Linear mixed-model regression analyses for long-term post-operative renal functiona

\begin{tabular}{|c|c|c|}
\hline \multicolumn{3}{|l|}{ Univariate Analysis } \\
\hline Variable & $\begin{array}{l}\text { Regression } \\
\text { Coefficient }\end{array}$ & $P$ value \\
\hline DU vs. EM & 2.1 & 0.31 \\
\hline NU vs. EM & -4.46 & 0.009 \\
\hline \multicolumn{3}{|l|}{ Multivariate Analysis } \\
\hline Variable & $\begin{array}{l}\text { Regression } \\
\text { Coefficient }\end{array}$ & $P$ value \\
\hline DU vs. EM & 1.28 & 0.6 \\
\hline NU vs. EM & -5.06 & 0.023 \\
\hline Age & 0.02 & 0.69 \\
\hline \multicolumn{3}{|l|}{ Race } \\
\hline Caucasian & -1.32 & 0.71 \\
\hline African-American & -3.56 & 0.4 \\
\hline Hispanic & -1.9 & 0.67 \\
\hline Other & -0.4 & 0.93 \\
\hline Gender & 1.24 & 0.35 \\
\hline \multicolumn{3}{|l|}{ Pathologic Stage } \\
\hline $\mathrm{Ta}$ & -1.93 & 0.62 \\
\hline $\mathrm{TI}$ & -2.12 & 0.63 \\
\hline $\mathrm{T} 2$ & 0.71 & 0.87 \\
\hline T3 & -1.6 & 0.69 \\
\hline $\mathrm{T} 4$ & 2.97 & 0.54 \\
\hline Solitary Kidney Status & -0.78 & 0.92 \\
\hline Pre-Operative Hydronephrosis & 3.53 & 0.023 \\
\hline
\end{tabular}

${ }^{\mathrm{a} A b b r e v i a t i o n s: ~ E M, ~ e n d o s c o p i c ~ m a n a g e m e n t ; ~ D U, ~ d i s t a l ~ u r e t e r e c t o m y ; ~ N U, ~}$ nephroureterectomy

\section{Discussion}

UTUC is a rare but potentially aggressive disease entity, and $\mathrm{NU}$ is considered the gold standard of surgical treatment. ${ }^{15}$ EM has traditionally been reserved for patients with a solitary kidney, bilateral disease, or that not amenable to radical surgery. ${ }^{13,16}$ However, several recent studies have suggested that conservative therapy can be utilized in lower-risk disease, including small, unifocal, low grade, and low stage lesions. ${ }^{13}$ Furthermore, NU may prevent certain patients from being eligible for adjuvant therapy. ${ }^{17,18} \mathrm{~A}$ minimally invasive approach may help the patient avoid radical surgery while achieving adequate oncologic outcomes. ${ }^{3}$

Our results show that EM offers superior preservation of kidney function compared to NU after both short- and long-term intervals. In the long-term analysis, this advantage is maintained even when taking into account factors such as patient age and solitary kidney status. These findings are consistent with those from an analysis by Fajkovic et al comparing $20 \mathrm{EM}$ to $178 \mathrm{NU}$ that showed post-op renal function was significantly better in the EM group. ${ }^{14}$ Thus, our findings suggest that the short-term renal functional benefit of EM to NU is sustained over the long-term period, as well.

We found no significant change in renal functional outcomes between EM and DU after both short- and long-term intervals. Previous studies have classified DU as a conservative surgical approach compared to NU. ${ }^{19,20}$ As such, it seems the extirpative qualities associated with DU do not affect its ability to preserve the renal unit and subsequent function. In addition, DU has been shown to have adequate cancer specific survival, recurrence free survival, and metastasis free survival in small, low stage (less than or equal to T2) distal ureteral lesions. ${ }^{19,21}$ Moreover, Jeldres et al concluded that indications for DU might even be extended to certain T3 or T4 lesions. ${ }^{22}$ Our results underscore the utility of DU as a conservative renal-sparing option.

Our results support the prima facie logic of kidney-sparing procedures versus $\mathrm{NU}$ - removing the entire renal unit has a greater impact on renal function than a kidney-sparing approach. EM has been advocated in the literature for lower-risk lesions in the presence of a normal contralateral kidney. ${ }^{11,15,23,24}$ With lower-risk UTUC, EM has favorable oncologic outcomes; Cutress et al., ${ }^{25}$ showed an estimated mean disease specific survival of 190 months in a sample of 73 patients. Pak et al., ${ }^{26}$ have shown that conservative EM of UTUC can be cost-effective in the long term compared to NU. However, EM requires strict adherence to post-op surveillance due to a higher likelihood of recurrence. ${ }^{27}$ There are also concerns about inaccurate staging, inaccurate grading, and incomplete resection with EM. ${ }^{14}$ Our findings support the use of EM in select patients due to its superiority to $\mathrm{NU}$ and equivalency to DU in preserving renal function.

To our knowledge, our study cohort represents the largest utilized for this type of analysis. Several limitations to our study should be acknowledged. Our analysis is retrospective, and is limited by the biases inherent therein. We considered UTUC to be any urothelial cancer above the bladder and compared post-operative renal function between these treatment modalities irrespective of the location of the cancer to gauge functional impact on this disease entity as a whole. The analysis performed did not specifically compare DU to NU to allow the primary focus to be the impact of EM on postoperative renal function. Due to the long length of our study period tracking back to the earliest records available at our institution, there were inconsistencies with data reporting regarding comorbidities and complications postprocedure, and thus we were unable to implement these factors into the analysis. Although our cohort featured a robust follow-up period of 20.8 months, larger randomized prospective studies are needed to corroborate these results.

\section{Conclusion}

EM provides superior post-operative eGFR preservation to NU and equivalent preservation compared to DU in the management of UTUC. In appropriately selected patients willing to adhere to a strict surveillance protocol, kidney-sparing procedures may allow for favorable renal functional outcomes while providing adequate oncological control.

\section{Acknowledgements}

None.

\section{Conflict of interest}

The author declares no conflict of interest. 


\section{References}

1. Smith AK, Stephenson AJ, Lane BR, et al. Inadequacy of biopsy for diagnosis of upper tract urothelial carcinoma: implications for conservative management. Urology. 2011;78(1):82-86.

2. Margulis V, Shariat SF, Matin SF, et al. Outcomes of radical nephroureterectomy: a series from the Upper Tract Urothelial Carcinoma Collaboration. Cancer. 2009;115(6):1224-1233.

3. Grasso M, Fishman AI, Cohen J, et al. Ureteroscopic and extirpative treatment of upper urinary tract urothelial carcinoma: a 15-year comprehensive review of 160 consecutive patients. BJU Int. 2012;110(11):1618-1626.

4. Zhang QL, Rothenbacher D. Prevalence of chronic kidney disease in population-based studies: systematic review. BMC Public Health 2008;8:117.

5. Ristau BT, Tomaszewski JJ, Ost MC. Upper tract urothelial carcinoma: current treatment and outcomes. Urology. 2012;79(4):749-756.

6. Fanari M, Serra S, De Lisa A. Conservative endoscopic retrograde treatment of upper urinary tract tumors: retrospective analysis of the last 105 cases. Urologia. 2011;78(3):233-235.

7. Elliott DS, Blute ML, Patterson DE, et al. Long-term follow-up of endoscopically treated upper urinary tract transitional cell carcinoma. Urology. 1996;47(6):819-825.

8. Tawfiek ER, Bagley DH. Upper-tract transitional cell carcinoma Urology. 1997;50(3):321-329.

9. Zincke H, Neves RJ. Feasibility of conservative surgery for transitional cell cancer of the upper urinary tract. Urol Clin North Am. 1984;11(4):717-724.

10. Johnson DE, Babaian RJ. Conservative surgical management for noninvasive distal ureteral carcinoma. Urology. 1979;13(4):365-367.

11. Johnson GB, Grasso M. Ureteroscopic management of upper urinary tract transitional cell carcinoma. Curr Opin Urol. 2005;15(2):89-93.

12. Nison L, Roupret M, Bozzini G, et al. The oncologic impact of a delay between diagnosis and radical nephroureterectomy due to diagnostic ureteroscopy in upper urinary tract urothelial carcinomas: results from a large collaborative database. World J Urol. 2013;31(1):69-76.

13. Cutress ML, Stewart GD, Zakikhani P, et al. Ureteroscopic and percutaneous management of upper tract urothelial carcinoma (UTUC) systematic review. BJU Int. 2012;110(5):614-628.

14. Fajkovic H, Klatte T, Nagele U, et al. Results and outcomes after endoscopic treatment of upper urinary tract carcinoma: the Austrian experience. World J Urol. 2013;31(1):37-44.
15. Roupret M. Conservative management of urothelial carcinomas of the upper urinary tract. Prog Urol. 2012;22(14):861-866.

16. Blute M. Endourology: management of upper tract genitourinary pathology. Curr Probl Urol. 1991;1:4-36.

17. Lane BR, Smith AK, Larson BT, et al. Chronic kidney disease after nephroureterectomy for upper tract urothelial carcinoma and implications for the administration of perioperative chemotherapy. Cancer. 2010;116(12):2967-2973.

18. Xylinas E, Rink M, Margulis V, et al. Impact of renal function on eligibility for chemotherapy and survival in patients who have undergone radical nephro-ureterectomy. BJU Int. 2013;112(4):453-461.

19. Colin P, Ouzzane A, Pignot G, et al. Comparison of oncological outcomes after segmental ureterectomy or radical nephroureterectomy in urothelial carcinomas of the upper urinary tract: results from a large French multicentre study. BJU Int. 2012;110(8):1134-1141.

20. Silberstein JL, Power NE, Savage C, et al. Renal function and oncologic outcomes of parenchymal sparing ureteral resection versus radical nephroureterectomy for upper tract urothelial carcinoma. $J$ Urol. 2012;187(2):429-434.

21. Bagrodia A, Kuehhas FE, Gayed BA, et al. Comparative analysis of oncologic outcomes of partial nephrectomy vs. radical nephroureterectomy in upper tract urothelial carcinoma. Urology. 2013;81(5):972-977.

22. Jeldres C, Lughezzani G, Sun M, et al. Segmental ureterectomy can safely be performed in patients with transitional cell carcinoma of the ureter. J Urol. 2010;183(4):1324-1329.

23. Elliott DS, Segura JW, Lightner D, et al. Is nephroureterectomy necessary in all cases of upper tract transitional cell carcinoma? Longterm results of conservative endourologic management of upper tract transitional cell carcinoma in individuals with a normal contralateral kidney. Urology. 2001;58(2):174-178.

24. Chen GL, Bagley DH. Ureteroscopic management of upper tract transitional cell carcinoma in patients with normal contralateral kidneys. J Urol. 2000;164(4):1173-1176.

25. Cutress ML, Stewart GD, Wells-Cole S, et al. Long-term endoscopic management of upper tract urothelial carcinoma:20-year single-centre experience. BJU Int. 2012;110(11):1608-1617.

26. Pak RW, Moskowitz EJ, Bagley DH. What is the cost of maintaining a kidney in upper-tract transitional-cell carcinoma? An objective analysis of cost and survival. $J$ Endourol 2009;23(3):341-346.

27. Adamis S, Varkarakis J. Minimally invasive approach in the management of upper- urinary-tract tumours. Scand J Urol Nephrol. 2011;45(6):381-387. 